\title{
Rare sugar D-psicose prevents progression and development of diabetes in T2DM model Otsuka Long-Evans Tokushima Fatty rats
}

This article was published in the following Dove Press journal:

Drug Design, Development and Therapy

17 January 2015

Number of times this article has been viewed

\author{
Akram Hossain ${ }^{1,2}$ \\ Fuminori Yamaguchi ${ }^{1}$ \\ Kayoko Hirose' \\ Toru Matsunaga ${ }^{3}$ \\ Li Sui ${ }^{\prime}$ \\ Yuko Hirata' \\ Chisato Noguchi' \\ Ayako Katagi' \\ Kazuyo Kamitori' \\ Youyi Dong' \\ Ikuko Tsukamoto ${ }^{4}$ \\ Masaaki Tokuda' \\ 'Department of Cell Physiology, \\ Faculty of Medicine, Kagawa \\ University, Ikenobe, Miki, Kagawa, \\ Japan; ${ }^{2}$ Research and Development, \\ Matsutani Chemical Industry Co., \\ Ltd., Kitaitami, Itami-Shi, Hyogo, \\ Japan; ${ }^{3}$ Division of Hospital Pathology, \\ Faculty of Medicine, Kagawa \\ University, Ikenobe, Miki, Kagawa, \\ Japan; ${ }^{4}$ Department of Pharmaco- \\ Bio-Informatics, Faculty of Medicine, \\ Kagawa University, Ikenobe, Miki, \\ Kagawa, Japan
}

Background: The fundamental cause of overweight and obesity is consumption of calorie-dense foods. We have introduced a zero-calorie sweet sugar, D-psicose (D-allulose), a rare sugar that has been proven to have strong antihyperglycemic and antihyperlipidemic effects, and could be used as a replacement of natural sugar for the obese and diabetic subjects.

Aim: Above mentioned efficacy of D-psicose (D-allulose) has been confirmed in our previous studies on type 2 diabetes mellitus (T2DM) model Otsuka Long-Evans Tokushima Fatty (OLETF) rats with short-term treatment. In this study we investigated the long-term effect of D-psicose in preventing the commencement and progression of T2DM with the mechanism of preservation of pancreatic $\beta$-cells in OLETF rats.

Methods: Treated OLETF rats were fed 5\% D-psicose dissolved in water and control rats only water. Nondiabetic control rats, Long-Evans Tokushima Otsuka (LETO), were taken as healthy control and fed water. To follow the progression of diabetes, periodic measurements of blood glucose, plasma insulin, and body weight changes were continued till sacrifice at 60 weeks. Periodic in vivo body fat mass was measured. On sacrifice, pancreas, liver, and abdominal adipose tissues were collected for various staining tests.

Results: D-Psicose prevented the commencement and progression of T2DM till 60 weeks through the maintenance of blood glucose levels, decrease in body weight gain, and the control of postprandial hyperglycemia, with decreased levels of $\mathrm{HbA}_{1 \mathrm{c}}$ in comparison to nontreated control rats. This improvement in glycemic control was accompanied by the maintenance of plasma insulin levels and the preservation of pancreatic $\beta$-cells with the significant reduction in inflammatory markers. Body fat accumulation was significantly lower in the treatment group, with decreased infiltration of macrophages in the abdominal adipose tissue.

Conclusion: Our findings suggest that the rare sugar D-psicose could be beneficial for the prevention and control of obesity and hyperglycemia with the preservation of $\beta$-cells in the progression of T2DM.

Keywords: rare sugar D-psicose, OLETF rats, type 2 diabetes mellitus, insulin resistance, adiposity, $\beta$-islet preservation

\section{Introduction}

Overweight and obesity are not only the leading risk factors for global deaths but also the major contributor to other serious diseases worldwide, including heart disease, cancers, and type 2 diabetes. Mainly because of the uncontrolled intake of high-fat and high-cholesterol diet, the prevalence of overweight and rate of obesity drastically increase. Following this, type 2 diabetes mellitus (T2DM) inevitably arises, which warrants attentive consideration of the vital role of food for prevention and treatment.
Correspondence: Akram Hossain of Medicine, Kagawa University, I750-I Ikenobe, Miki, Kagawa, 76I-0793, Japan

Tel +81878912095

Fax +8I 87 89। 2096

Email hossain@med.kagawa-u.ac.jp 
The inextricably interlinked overweight, obesity, and T2DM, usually commencing from obesity, if not arrested, might be associated with life-threatening consequences of cardiac and metabolic diseases, high blood pressure, atherosclerosis, and T2DM. ${ }^{1}$ Moreover, chronic intake of high calories forces the pancreatic $\beta$-cells to produce more insulin in order to cope with body demands to maintain glucose homeostasis, which, in turn, increases $\beta$-cell function and mass. ${ }^{2}$ When the pancreas fails, insulin resistance becomes decompensated and hyperglycemia is detected. Among the leading mechanisms of insulin resistance, nonresolving low-grade inflammation that involves a number of inflammatory cytokines, ${ }^{3}$ lipids and their metabolites, and reactive oxygen species (ROS) have been mentioned. ${ }^{4}$ It is found that macrophages accumulate in inflammation tissue and discharge cytokines ${ }^{3}$ such as proinflammatory cytokines, interleukin 6 (IL-6), interleukin (IL-1 $\beta$ ), and tumor necrosis factor $\alpha($ TNF- $\alpha)$. Obesity itself is also closely related to systemic adipose inflammation with aggregation of macrophages, and high levels of those cytokines from them aggravate altogether insulin resistance with the progression of T2DM. Although regular exercise and low-calorie diet could improve hyperglycemia and associated complications related to T2DM, most patients need medication. Although some antihyperglycemic products can improve the interrelated complications, they cause weight gain and, in turn, be associated with high blood pressure and dyslipidemia. Pharmacological approaches such as sulfonylureas and metformin have not been able to adequately improve the consequences of insulin resistance so far.

Therefore, attention has been focused on the study of herbal medicines that may not only provide better protection with lesser side effects but also ameliorate the associated comorbidities with the prevention of $\beta$-cell failure. ${ }^{5}$ Additionally, with the intense attention of researchers and the growing interest of the general public concerning the treatment and prevention of diseases, functional foods have been explored. ${ }^{6}$ It has been shown that functional foods contribute to the improvement of overall health and reduce the occurrence of diseases. ${ }^{7}$ Recently, in an attempt to produce functional foods aimed at low calorie and less sugar intake, various foods with D-psicose added as a substitute for sugar have been prepared and marketed locally as FOSHU (Food for Specific Health Use).

D-Psicose (D-allulose), one of the rare sugars, has been proven to have antihyperglycemic, antihyperlipidemic, and anti-inflammatory effects. Owing to less availability, the biological functions of D-psicose have not been sufficiently explored. However, the innovation of a unique production method developed by Izumori ${ }^{8}$ enabled a number of investigations. Studies have shown that D-psicose is effective experimentally against obesity and T2DM in normal ${ }^{9,10}$ and in T2DM rat models, ${ }^{11-13}$ and clinically in healthy ${ }^{14}$ and borderline diabetic humans. ${ }^{15}$ Thus, these studies reflect the commercial use of D-psicose as a sugar substitute against hyperglycemia and hyperlipidemia. The possible mechanisms of controlling high glucose levels, such as potency of absorption of D-psicose over D-glucose from the intestine ${ }^{16}$ and inhibition of the activities of enzymes glucoamylase and maltase, have been investigated. ${ }^{10}$ Our previous study showed that D-psicose could play a role in the metabolic regulation in T2DM OLETF (Otsuka Long-Evans Tokushima Fatty) rats through maintaining blood glucose levels and preventing fat accumulation in the abdomen. ${ }^{12}$ The possible mechanisms to maintain hyperglycemia may be through the translocation of glucokinase activity, which has been shown to be lower in hyperglycemic or diabetic condition, ${ }^{17}$ as well as through the protection of the pancreatic $\beta$-cells from injury caused by hyperglycemia. ${ }^{13}$

D-Psicose has also been shown to inhibit hepatic fatty acid synthase as the mechanism of fat control, followed by decreased body weight gain, when compared to D-glucose. ${ }^{18}$ In addition to the antihyperglycemic and antihyperlipidemic effects of D-psicose, its zero-calorie credit and $70 \%$ relative sweetness ${ }^{9}$ also attracted some of the food companies to produce various foodstuffs containing D-psicose. US Food and Drug Administration (FDA) recognized D-psicose as Generally Recognized as Safe (GRAS) and has permitted its use as a food ingredient, with GRN Number 400. ${ }^{19}$

In our previous studies, ${ }^{12,13}$ we fed OLETF rats with D-psicose for 3 months till the age of 20 weeks, around when these rats usually develop mild hyperglycemia. ${ }^{20}$ However, in this study we continued D-psicose feeding till the age of 60 weeks in order to study several issues such as whether D-psicose could maintain prolonged sustained normoglycemia; protect $\beta$-cells against severe damage observed at this stage; control the cytokines related to inflammation since amelioration of oxidative stress and modulation of proinflammatory parameters have been shown to be beneficial for T2DM patients. ${ }^{21}$

\section{Materials and methods}

\section{Animals, drugs, foods, and drinks}

Both OLETF and LETO (Long-Evans Tokushima Otsuka) rats were bought from Otsuka Pharmaceutical Co. (Tokushima, Japan) at 4-week age and were allowed to adapt for 2 weeks. 
The animals were maintained at $23^{\circ} \mathrm{C} \pm 2^{\circ} \mathrm{C}$ and $55 \% \pm 5 \%$ humidity with 12-hour light/dark cycle. D-Psicose (D-allulose or D-ribo-2-hexulose; molecular formula, $\mathrm{C}_{6} \mathrm{H}_{12} \mathrm{O}_{6}$; molecular weight, 180.156) is a rare ketohexose, named "rare sugar," supplied by Kagawa University Rare Sugar Research Center (Miki, Kagawa, Japan). Rats were fed MF rat chow (Oriental Yeast Company Limited, Tokyo, Japan). D-Psicose was dissolved in normal tap water, and the rats were allowed to drink freely.

\section{Experimental protocol}

Six-week-old OLETF rats were divided into 2 groups $(n=10$ each): OLETF psicose (O-P) and OLETF control (O-C). Psicose group was given 5\% D-psicose; O-C and LETO were given water only. All rats were supplied MF food pellets and their respective drinks. Body weight was measured every week till 60 weeks. Food and drink intakes were measured for three consecutive days each week and the average rat/ day intake was calculated. Periodic fasting and postprandial blood glucose levels were measured using a freestyle glucose meter (YSI 2300-STAT) from tip of the tail. Plasma was collected from the blood by centrifugation and was stored at $-80^{\circ} \mathrm{C}$ until analysis. At the end of 60 weeks, the rats were fasted for 12 hours and then anaesthetized deeply. Then, the abdomen was opened, blood was withdrawn, and necessary organs were removed, weighed, and preserved as per measurements and staining procedures. Abdominal fat was collected from the epididymal, retroperitoneal, and mesenteric spaces and weighed.

\section{Tests and measurements}

Rats were fasted 12 hours for oral glucose tolerance test (OGTT), 0-minute sample was collected, and then each rat was fed $2 \mathrm{~g} / \mathrm{kg}$ body weight glucose by gavage and blood was collected at 30, 60, 90, and 120 minutes after gavage. The frozen plasma was used to measure insulin and lipids (total cholesterol [TC], triglycerides [TG], and high- and low-density lipoproteins [HDL and LDL]). Serum levels of glutathione (GSH), IL-6, TNF- $\alpha$, leptin, and adiponectin were measured using rat-specific enzyme-linked immunosorbent assay (ELISA) kits (R\&D Systems Inc, Minneapolis, MN, USA) following manufacturer's recommendations. For in vivo body composition measurement, bioimpedance spectroscopy (ImpediVet, ImpediMed Ltd., Brisbane, QLD, Australia) was used to estimate total fat mass (FM), fat-free body mass, and body mass index (BMI). ${ }^{22}$

To evaluate insulin resistance severity, homeostasis model assessment (HOMA) was calculated. ${ }^{23}$ The details of this calculation are mentioned in our previous study. ${ }^{13}$ To measure the inflammatory profile, the pancreas and adipose tissues were fixed in formalin, embedded in paraffin, and stained with hematoxylin and eosin (HE). Light microscopy was used to evaluate adipocyte histology, and ImageJ (NIH, Bethesda, MD, USA) software was used to count the pancreas islet number, but it was very difficult to identify clearly because of the disorganized and broken status of the islets of Langerhans. Hence, the islets were counted after insulin immunostaining. However, the islet cell mass was calculated by multiplying $\beta$-cell percentage by total pancreatic weight and the percentage by insulin-positive staining. For immunostaining of insulin and glucagon, the pancreas tissue was embedded in optimal cutting temperature compound and stained with respective antibodies (Funakoshi, Japan).

\section{Statistics}

All values presented are the mean \pm standard deviation (SD). Significant difference among the groups was determined by one-way analysis of variance (ANOVA), and differences between groups were analyzed by the post hoc test Hoechberg's for equal and Games-Howell for unequal variances by using the SPSS software (version 17.0, SPSS, Chicago, IL, USA). A $P$-value of $<0.05$ was taken as significant.

\section{Results Antihyperglycemic effect of D-psicose}

Changes in blood glucose and serum insulin concentration: Fasting blood glucose levels increased gradually in the OLETF groups and were significantly higher all through the experimental period as compared with those in nondiabetic LETO group (Figure 1A). In the O-C group, the glucose levels started to increase slowly from 25 weeks and then sharply till 60 weeks, whereas in the O-P group the glucose levels started to increase slightly from 45 weeks and remained constant till 60 weeks (Figure 1A). In contrast, glucose levels in LETO group remained unchanged until 60 weeks. The rise of blood glucose levels between O-C and O-P groups was significant from 35 weeks $(P<0.01)$ till sacrifice. Periodic fasting serum insulin levels increased gradually from week 10 in the O-C group, peaked at 30 weeks, and decreased thereafter, even decreasing to almost zero level at week 60 (Figure 1B). These increases from weeks 20 to 40 were significantly higher than those in both O-P and LETO groups. In contrast, plasma insulin levels in both O-P and LETO groups increased slightly from week 10 and then remained almost unchanged until week 60 . Glucose levels from the oral glucose tolerance test were significantly higher 
A

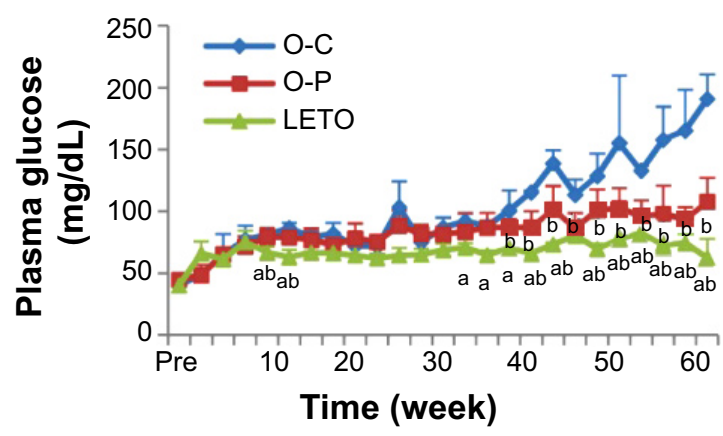

B

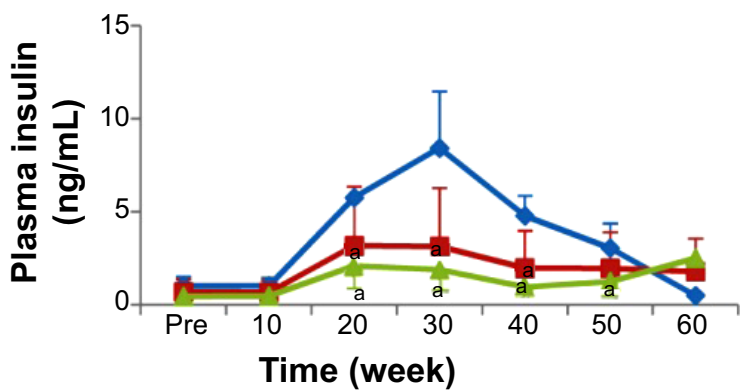

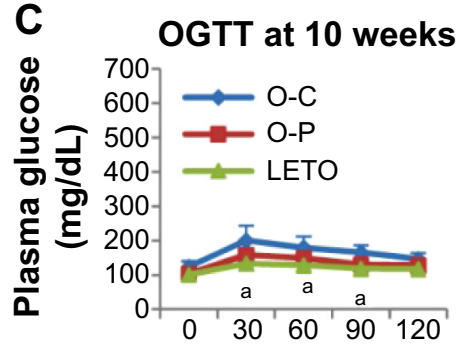

Time (minutes)

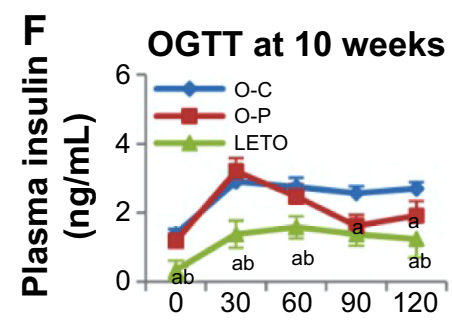

Time (minutes)

I

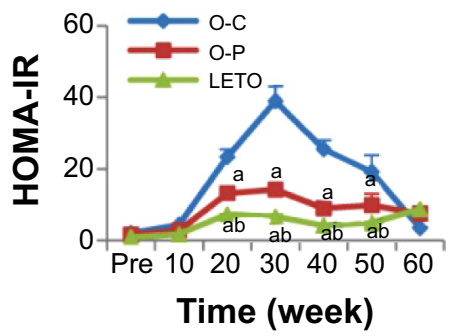

D

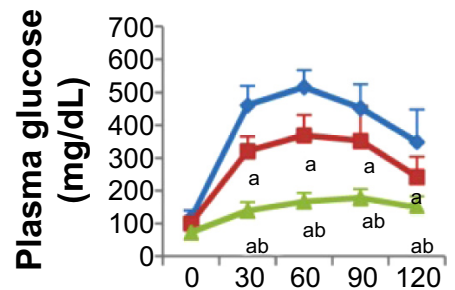

Time (minutes)

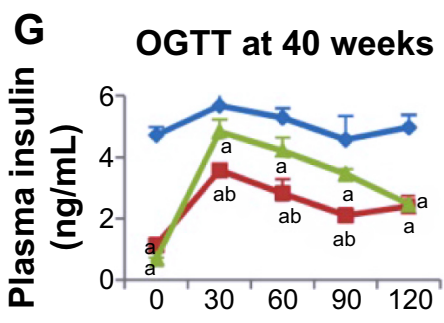

Time (minutes)

J

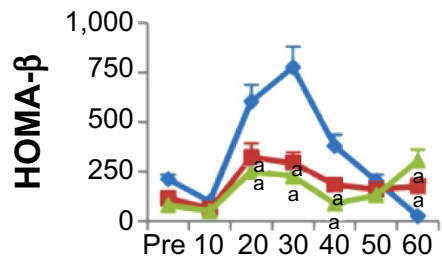

Time (week)

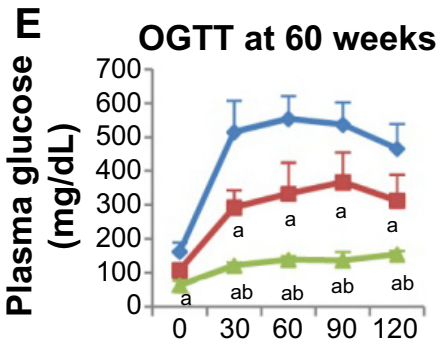

Time (minutes)

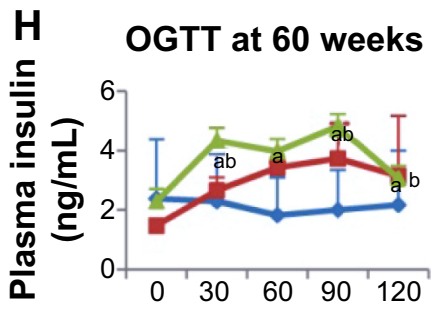

Time (minutes)

K

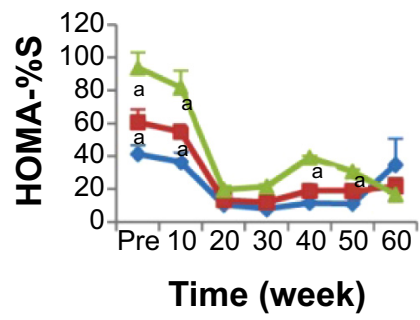

L

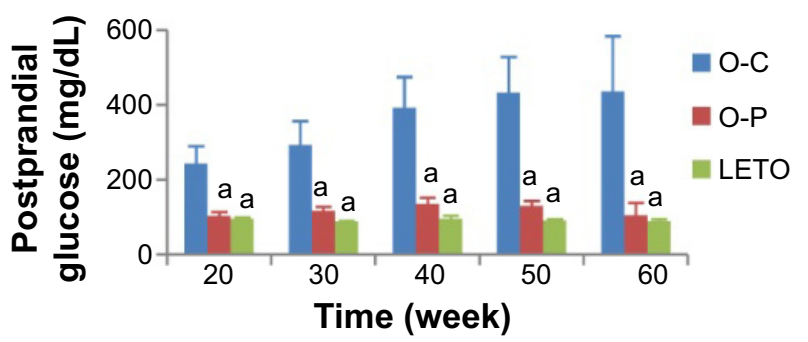

M

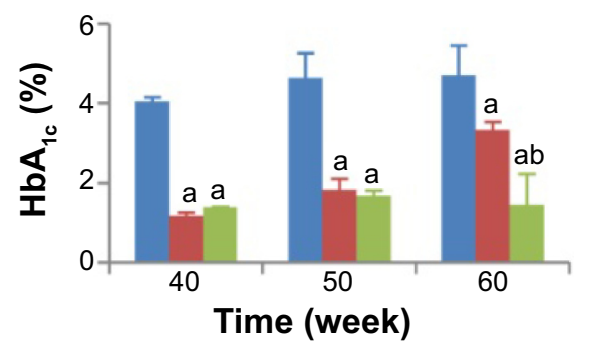

Figure I Periodic changes in fasting blood glucose (A) and insulin (B) concentrations with long-term treatment with or without rare sugar D-psicose in rats till age 60 weeks. Notes: The O-P group was treated with $5 \%$ D-psicose dissolved in drinking water and all the rats were supplied with a standard rat diet. (C-E) show changes in blood sugar and $(\mathbf{F}-\mathbf{H})$ show plasma insulin levels during 2 -hour oral glucose $(2 \mathrm{~g} / \mathrm{kg}$ body weight in $50 \%$ solution) tolerance test. (I-K) show serial changes of HOMA-IR, HOMA-\% $\beta$, and HOMA-\%S. (L) shows changes in postprandial blood glucose levels, and (M) shows $\mathrm{HbA}$, levels. ${ }^{2}$ Represents significance versus $\mathrm{O}-\mathrm{C}$ group. ${ }^{\mathrm{b}} \mathrm{Represents}$ significance versus O-P group. Values are given as mean \pm SD ( $n=9$ for OLETF rats and $n=6$ for LETO rats).

Abbreviations: $\mathrm{HbA}_{\mathrm{Ic}}$, hemoglobin $\mathrm{A}_{\mathrm{Ic}}$; HOMA, homeostasis model assessment; LETO, Long-Evans Tokushima Otsuka; OGTT, oral glucose tolerance test; OLETF, Otsuka Long-Evans Tokushima Fatty; O-C, OLETF control; O-P, OLETF psicose. 
in the $\mathrm{O}-\mathrm{C}$ group in all time points at every week than those in the O-P group. And the levels in the LETO group were significantly lower than in the O-C group at every week and the O-P group at weeks 40 and 60 (Figure 1C-E). Initially, at week 10 blood glucose levels returned close to base line 120 minutes after gavage in all groups, whereas the return values in 120 minutes were much higher in both O-C and O-P groups at week 40 and markedly higher in the O-C group at week 60. At week 40, plasma insulin levels in the O-C group were significantly higher than in O-P group in all time points, including 0 minutes, whereas at week 60 the levels became significantly lower than those in the O-P group (Figure 1F-H).

Both HOMA-IR and HOMA- $\beta$ indexes were significantly higher in the O-C group than in both O-P and LETO groups, indicating the state of insulin resistance in O-C rats in which $\beta$-cells attempted to cope with increased insulin demand. In the O-P group the levels of HOMA-IR and HOMA- $\beta$ were decreased and HOMA- $\% \mathrm{~S}$ (insulin sensitivity) was consistently increased (Figure 1I-K).

Postprandial blood glucose levels elevated significantly in the O-C group gradually at weeks 20 and $30(P<0.01)$ and then markedly till week $60(P<0.001)$ than in both O-P and LETO groups, whereas there was no difference between O-P and LETO groups (Figure 1L). The levels of $\mathrm{HbA}_{1 \mathrm{c}}$ were significantly higher in the O-C group than in both O-P and LETO groups; however, the O-P group also showed a significantly higher value than that in LETO group at week 60, which was still significantly lower than that in O-C group (Figure 1M).

\section{Antiobesity effect of D-psicose}

Body weight, and food and drink consumption: Although the body weight of both the OLETF groups increased progressively with age, the body weight of all individual O-C rats was higher than that of all individual O-P rats till 33 weeks of age, and thereafter, 5 out of 9 (56\%) O-C rats started to show a decline in body weight but none $(0 \%)$ in the O-P group (Figure 2A, B). At 50 weeks, one more rat in the O-C group showed a decline in body weight (Figure 2A) and the rest of the 3 rats continued to gain body weight slowly till 55 weeks and finally started to decline till 60 weeks. In contrast, with long-term D-psicose treatment, the O-P rats continued to gain body weight spontaneously with age like healthy rats till 60 weeks (Figure 2B). However, the average weight gain in the O-P group was significantly lower than that in the O-C group (Figure 2C).

D-Psicose decreased food intake, with the average daily intake of the O-C, O-P, and LETO groups being 33.90 \pm 8.40 , $26.11 \pm 7.02$, and $22.63 \pm 0.34 \mathrm{~g} / \mathrm{rat} / \mathrm{d}$, respectively (Figure 2D).
Drink consumption was also significantly lower in the O-P group than in the O-C group from week 35 (Figure 2E).

Plasma lipids, body composition, and FM: Fasting plasma concentration of TC in OLETF groups increased significantly from week 30 than that in the LETO group, and among the OLETF groups, TC level was significantly lower in the O-P group than in the O-C group at 50 and 60 weeks (Figure 3A). There was no significant difference in TG levels except at week 60 (Figure 3B). Percent FM was lower in the O-P group than in the O-C group, significantly at week 30 and nonsignificantly at week 60 (Figure 3C). However, FM was significantly lower with corresponding high fat-free body mass in LETO than in OLETF rats. Body mass index was significantly higher in the O-C group than in both O-P and LETO groups (Figure 3D). Plasma lipoproteins were significantly higher in OLETF groups than in LETO from 30 weeks onward, and significantly higher levels of HDL at weeks 40 and 60 and LDL at weeks 50 and 60 in the $\mathrm{O}-\mathrm{C}$ than in the O-P (Figure $3 \mathrm{E}$ and $\mathrm{F}$ ) were observed. The amount of fat deposits (mesenteric + epididymal + retroperitoneal) (Figure 3G) and the number of large adipocytes (Figure $3 \mathrm{H}$ ) were significantly lower in the O-P group than in the $\mathrm{O}-\mathrm{C}$ group, whereas the total number of adipocytes in the O-P group was significantly higher than that in the O-C group (Figure 3I). Representative H\&E-stained photomicrographs of adipocytes are presented as O-C (Figure 3J), O-P (Figure 3K), and LETO (Figure 3L).

\section{Anti-inflammatory and $\beta$-cell-preserving effects of D-psicose}

In light microscopic findings, $\mathrm{O}-\mathrm{C}$ rats showed striking hyperplastic changes in the islets of Langerhans with extensive fibrosis. Most of the islets were disorganized, fibrotic, separated into small clusters, resulting in multinodular islets which were separated by connective tissue bands. As a result, the number of islet cells was decreased (Figure 4, adjacent table). Fatty degeneration was pronounced in the hypertrophied islets. These histological alterations were prominent and severe in the $\mathrm{O}-\mathrm{C}$ group (Figure 4A) in comparison to the O-P group (Figure 4B). In the O-C group, large or medium-size islets were hardly observed, while many were seen in both the O-P and the LETO groups. In the LETO group, these histological changes were not observed, except for some fatty degeneration (Figure 4C). To elucidate the islet architecture, better immunofluorescence studies using anti-insulin and antiglucagon antibodies were performed. The pattern of $\beta$-cells in the islets of LETO rats was normal, with $\beta$-cells (red color) located centrally and $\alpha$-cells (green color) at the periphery (Figure 4F). 

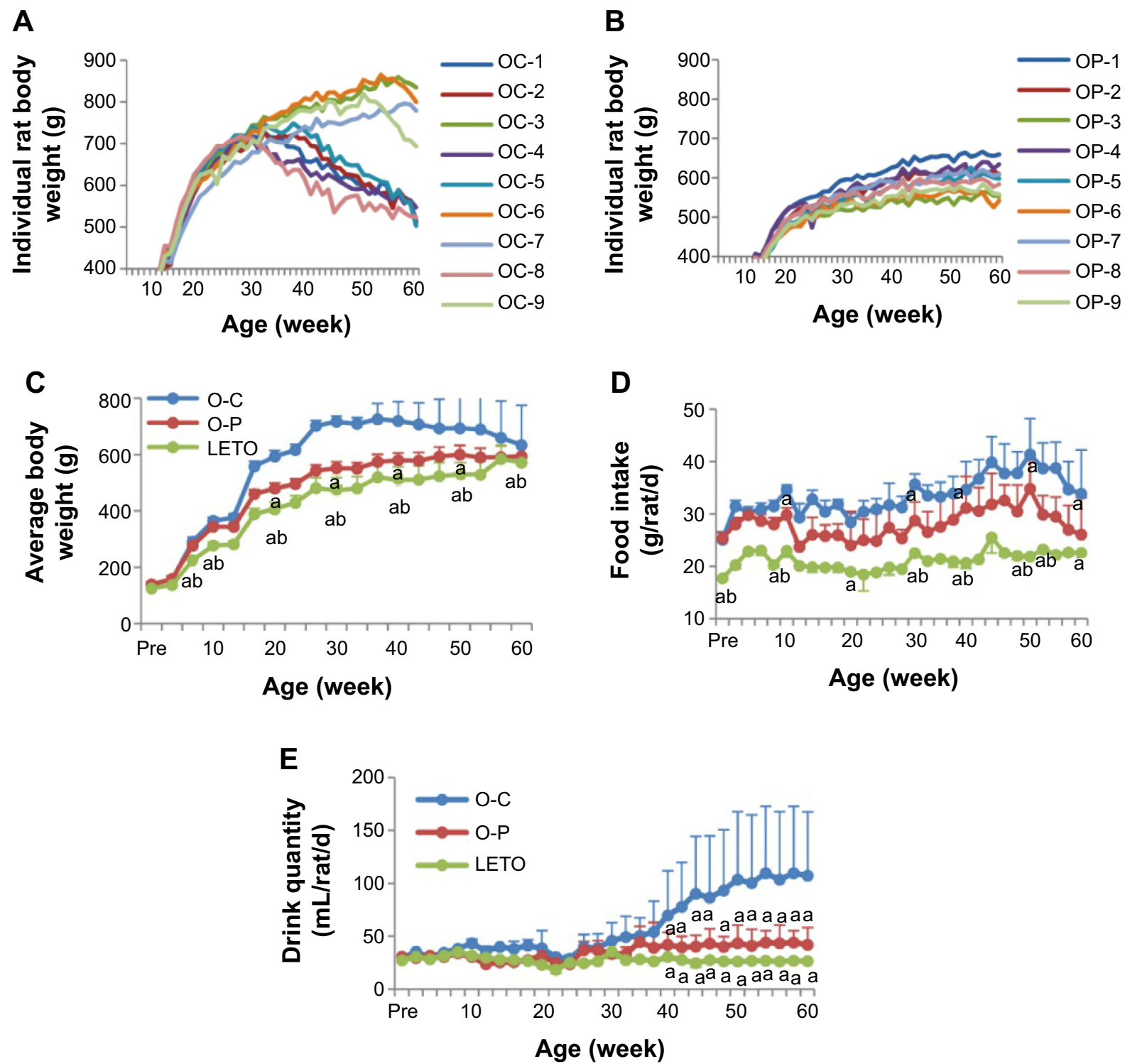

Figure 2 Periodic changes in individual rat body weight of O-C (A) and O-P (B) groups with long-term treatment with or without rare sugar D-psicose in rats till age 60 weeks.

Notes: The O-P group was treated with 5\% D-psicose dissolved in drinking water, where O-C and non-diabetic LETO were fed drinking water only. All the rats were supplied a standard rat diet. Changes in average body weight (C), quantity of average food intake (D), and average drink intake (E). ${ }^{2}$ Represents significance versus $O-C$ group.

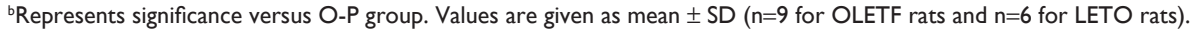

Abbreviations: LETO, Long-Evans Tokushima Otsuka; OLETF, Otsuka Long-Evans Tokushima Fatty; O-C, OLETF control; O-P, OLETF psicose.

However, the distribution was changed to a mixed pattern at week 60 in the O-C group (Figure 4D), where many of the insulin-positive cells lost their normal structure and were seen as a mixture of single or group of cells distributed all over the pancreas. This scattered distribution was rarely observed in the O-P group (Figure 4E) and not at all in the LETO group. The replacement of insulin-containing granules in $\beta$-cells by fat droplets and the distorted or diminished boundary of islets were very prominent in the O-C group (Figure 4A and D).

Plasma levels of leptin were elevated significantly in the O-C group than in both O-P and LETO groups from 20 weeks of age (Figure 4G), although there was no significant difference in plasma adiponectin levels among the groups (Figure 4H). As for the inflammatory cytokines, significantly high levels of both TNF- $\alpha$ and IL-6 were observed in the O-C group than in both O-P and LETO groups (Figure 4I and J). Plasma GSH was significantly high in the LETO group at 30 weeks of age, whereas these high levels decreased to trace amounts at 60 weeks (Figure 4K), although there was no significant difference among the groups at week 60 .

In relation to adipocyte inflammation, immunostaining of macrophage-expressed CD68 and F4/80 in adipose tissue was performed and an increased expression of both CD68 (Figure 5A-C) and F4/80 (Figure 5D-F) was found in the O-C group than in both O-P and LETO groups. 
A
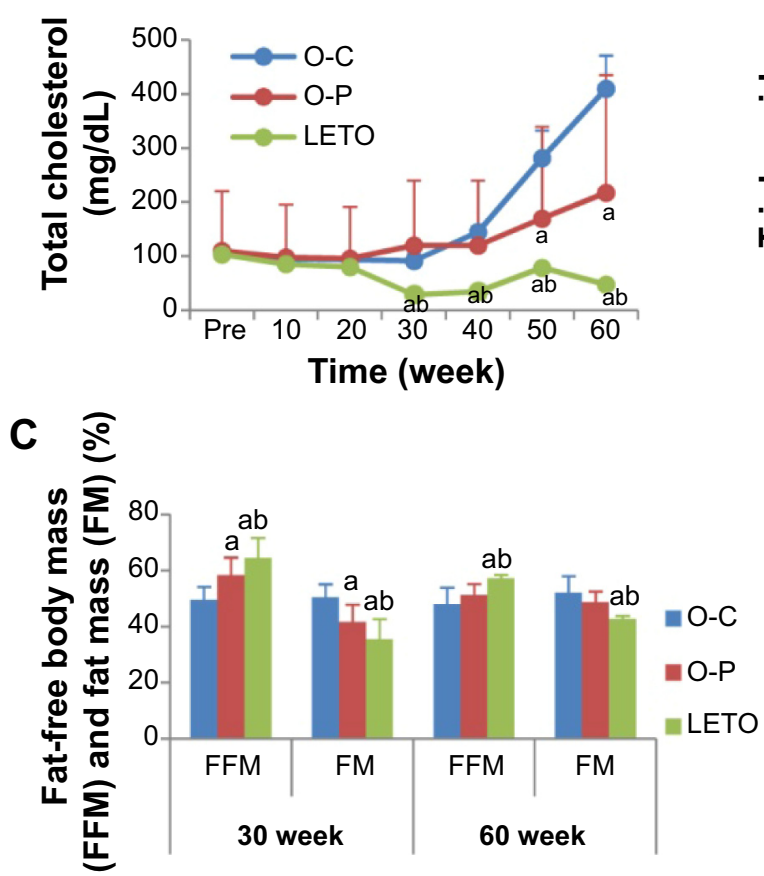

E

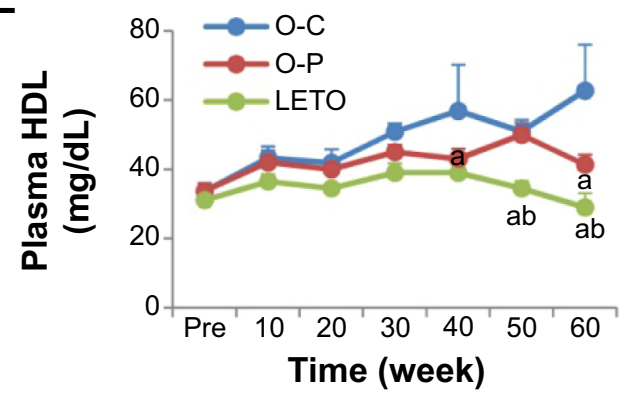

B

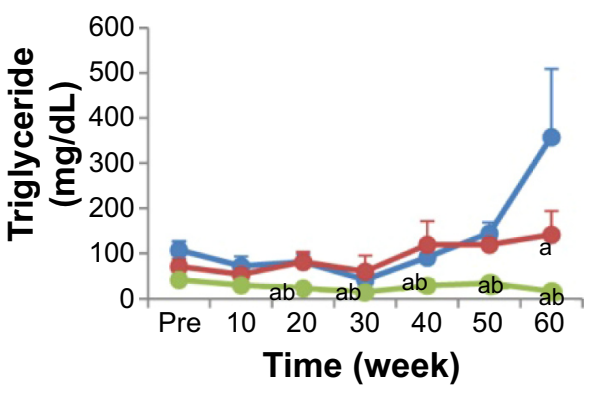

D

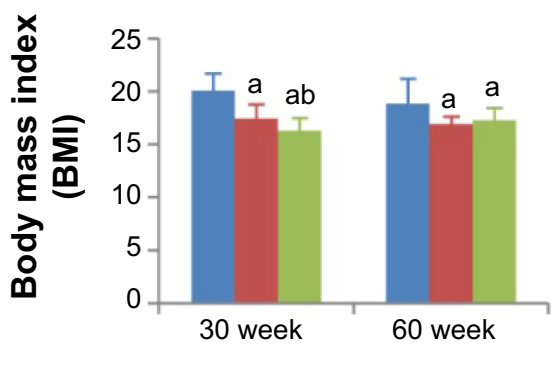

$\mathbf{F}$

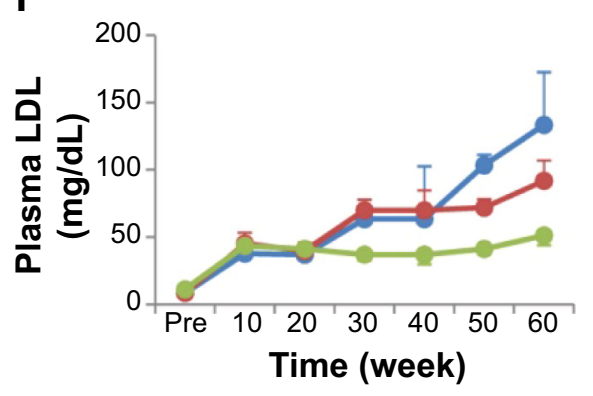

G

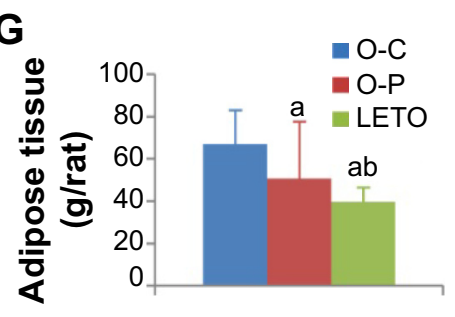

H

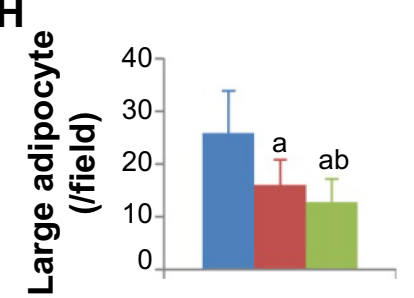

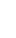

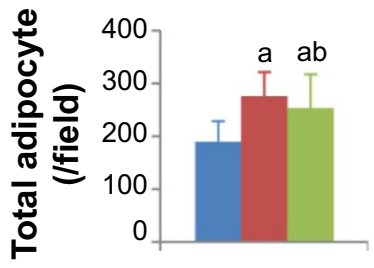

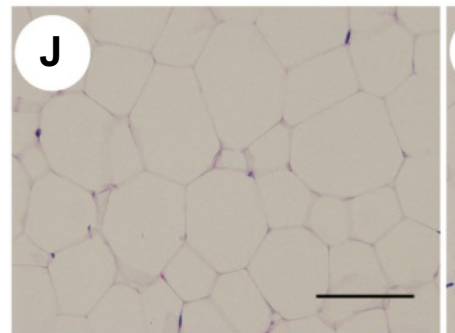
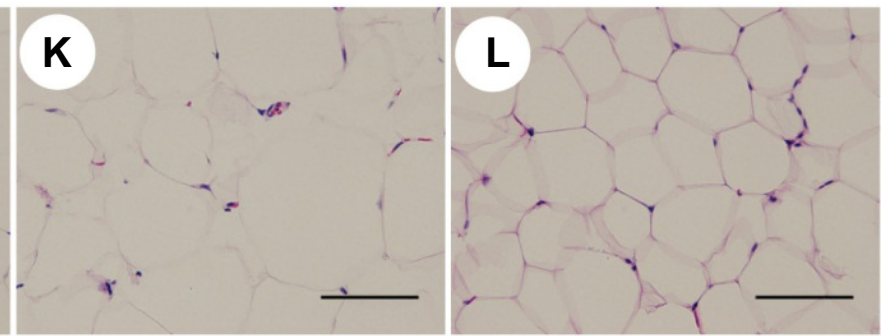

Figure 3 Periodic changes of blood lipid profile in rats of all groups with long-term treatment with or without rare sugar D-psicose till age 60 weeks.

Notes: The O-P group was treated with 5\% D-psicose dissolved in drinking water, where O-C and non-diabetic LETO were fed drinking water only. All the rats were supplied standard rat diet. Changes in serum total cholesterol (A), triglyceride (B), FFM and FM (C), BMI (D), HDL (E), and LDL (F) were shown. Data in (G-I) represent wet weight of adipose tissue, number of large adipocytes, and number of total adipocytes, respectively. (J-L) represents $\mathrm{HE}$ staining of adipose tissue of $\mathrm{O}-\mathrm{C}$ (J), O-P (K), and LETO (L) at age 60 weeks. ${ }^{a}$ Represent significance versus O-C group. ${ }^{b}$ Represents significance versus O-P group. Values are given as mean \pm SD ( $n=9$ for OLETF rats and $\mathrm{n}=6$ for LETO rats). Magnification: $\times 200$. Scale bars: $100 \mu \mathrm{m}$.

Abbreviations: BMI, body mass index; FM, fat mass; FFM, fat-free mass; HDL, high-density lipoprotein; LDL, low-density lipoprotein; LETO, Long-Evans Tokushima Otsuka; OLETF, Otsuka Long-Evans Tokushima Fatty; O-C, OLETF control; O-P, OLETF psicose; HE, hematoxylin and eosin. 

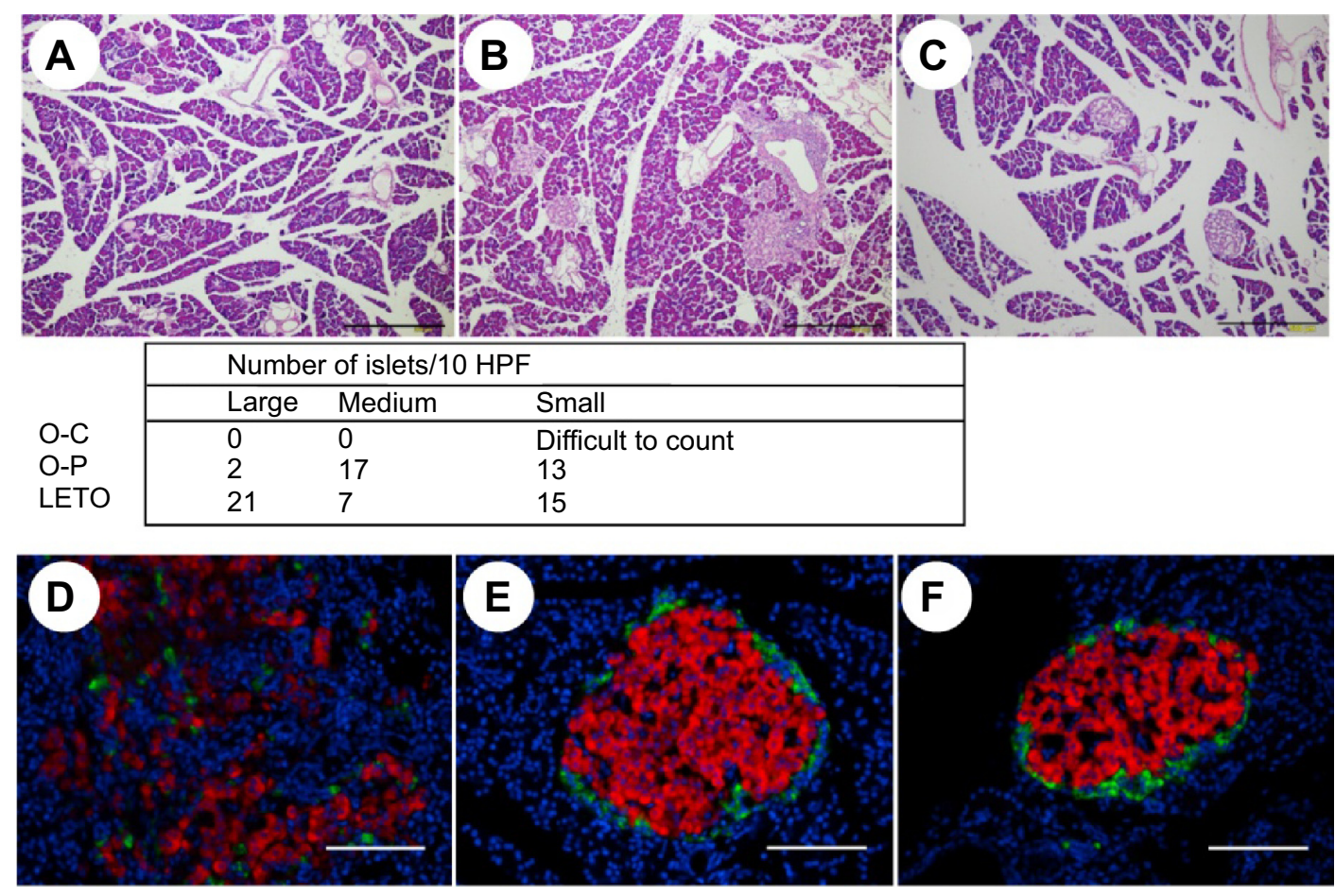

G

H
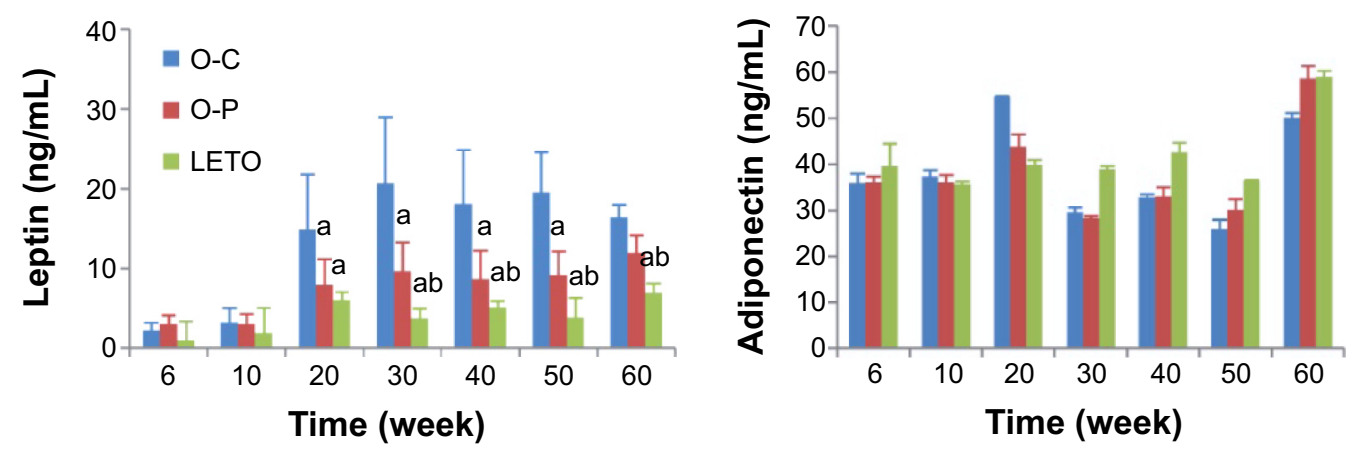

I

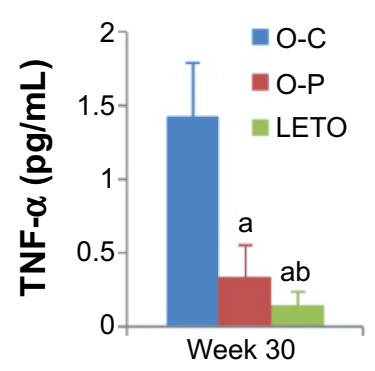

$\mathbf{J}$

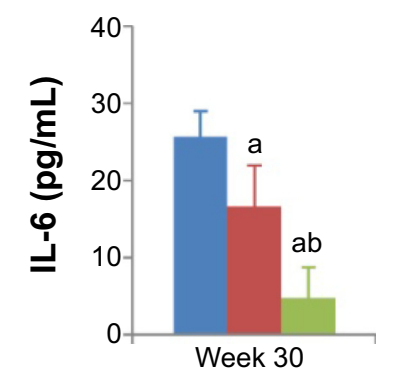

K

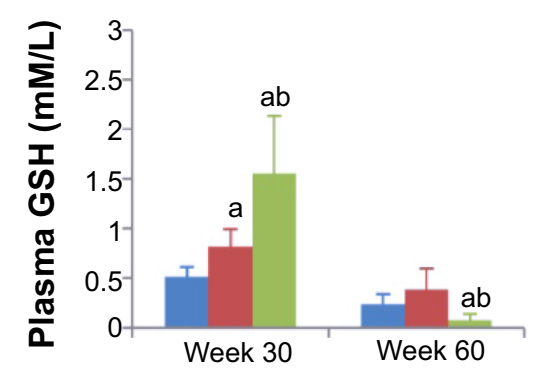

Figure 4 Changes in light microscopic features of pancreas of rats of all groups at week 60 with long-term treatment with or without rare sugar D-psicose.

Notes: The O-P group was treated with 5\% D-psicose dissolved in drinking water, where O-C and non-diabetic LETO were fed drinking water only. All the rats were supplied a standard rat diet. Changes of the pancreas of O-C (A), O-P (B), and LETO (C) rats stained with HE at age 60 weeks during sacrifice. (A) Islet disorganization with severe fibrosis, atrophy, and markedly infiltrated inflammatory cells. (B) Some islets were enlarged, but minimal fibrosis and infiltration of inflammatory cells were observed in the pancreas in D-psicose-treated rats. (C) Histological changes were absent or rarely observed in the pancreas of nondiabetic control LETO rats. The table adjacent to the HE pictures depicts the number of different-sized islets in all groups, with almost absent intact large and medium-size islets in the O-C group in comparison to O-P and LETO groups. (D-F) show immunofluorescence staining for insulin (red color) stained with anti-insulin antibody and glucagon (green color) stained with antiglucagon antibody to identify the presence and organization of both $\beta$ - and $\alpha$-cells in the pancreas at age 60 weeks. $(\mathbf{G}, \mathbf{H})$ Serial changes of serum levels of leptin and adiponectin,

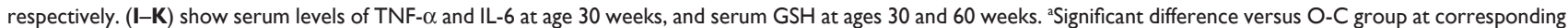
age. 'Significant difference versus O-P group at corresponding age. Values are expressed as mean \pm SD ( $n=9$ for OLETF rats and $n=6$ for LETO rats). Magnification: $\times 40$; scale bars: $500 \mu \mathrm{m}$ (Figures A-C). Magnification: $\times 200$; scale bars: $100 \mu \mathrm{m}$ (Figures D-F).

Abbreviations: GSH, glutathione; HPF, high power field; IL-6, interleukin 6; LETO, Long-Evans Tokushima Otsuka; OLETF, Otsuka Long-Evans Tokushima Fatty; O-C, OLETF control; O-P, OLETF psicose; TNF, tumor necrotic factor; HE, hematoxylin and eosin. 


\section{Discussion}

Our study distinctly demonstrated that long-term treatment with D-psicose until 60 weeks exerted beneficial effects against the progression of the sequences of T2DM and pancreatic $\beta$-cell damage in OLETF rats. Onset of T2DM occurs on failure of $\beta$-cells, and among the proposed mechanisms of $\beta$-cell failure, glucotoxicity, lipotoxicity, oxidative stress, ER stress, and amyloid deposition ${ }^{24}$ were mentioned, and almost all these factors may induce islet inflammation.

Severe damage with marked $\beta$-cell loss in the nontreated O-C animals was observed with $\mathrm{H} \& \mathrm{E}$ staining of the pancreas, and the damage was consistent with the high levels of both serum IL- 6 and TNF- $\alpha$. The levels of cytokines usually rise in insulin-resistant states, such as obesity, ${ }^{25}$ impaired glucose tolerance, ${ }^{26}$ and T2DM. ${ }^{27}$ The most important source of both pro- (IL-6, TNF- $\alpha$ ) and anti-inflammatory (adiponectin) cytokines is visceral adipose tissue, ${ }^{28}$ the aggregation of which was significantly reduced in the animals treated with D-psicose (Figure 3G). We think the inflammatory damage of $\beta$-cells was related to increased levels of proinflammatory cytokines in the control animals, which, in turn, contributed to the development of insulin resistance and T2DM. As shown in Figure $4 \mathrm{~A}-\mathrm{F}$, the destructive damage of the islets was observed in OLETF control rats but not in D-psicose-treated OLETF rats at 60 weeks of age. Of course, no pathological damage was observed at 10 weeks of age in all groups, since diabetic onset usually starts around 20 weeks of age. Therefore, it is assumed that the improvement of insulin resistance and metabolic syndrome by D-psicose treatment was facilitated through the control of proinflammatory cytokines after the onset of diabetes in OLETF rats. A study has shown that obesity and obesity-related metabolic disturbances were entangled with elevated lowgrade noninfectious inflammation and oxidative stress. ${ }^{29}$ This obesity-associated inflammation originates mainly in visceral white adipose tissue. ${ }^{30}$ Also, proinflammatory cytokine IL-6 is released from inflamed adipocytes, which in turn activate macrophages to release another cytokine, TNF- $\alpha .{ }^{31}$ Our results demonstrated that D-psicose treatment exhibited significantly less macrophage infiltration in comparison to nontreated control rats (Figure 5), which in turn resulted in decreased serum levels of both IL- 6 and TNF- $\alpha$ (Figure 4I and J). Hence, we could conclude that the antiinflammatory effect of D-psicose may indirectly suppress the release of proinflammatory cytokines.

Additionally, D-psicose also exerted its anti-inflammatory effects through the significant decrease in serum leptin levels and thus contributed to the improvement of insulin resistance, although serum adiponectin levels were not altered. Accumulation of macrophages in adipose tissue is closely correlated with weight gain and insulin resistance in both rodents and humans, ${ }^{32}$ and reduction of macrophage content correlates with weight loss. ${ }^{3}$ The restoration of body weight by D-psicose (Figure 2B) might be considered to be due to the improvement of insulin resistance through the control of glucose. D-Psicose prevented excess fat accumulation, confirmed by decreased in vivo FM (Figure 3C) as well as abdominal fat weight (Figure 3G), with the significant reduction in body mass index (Figure 3D).

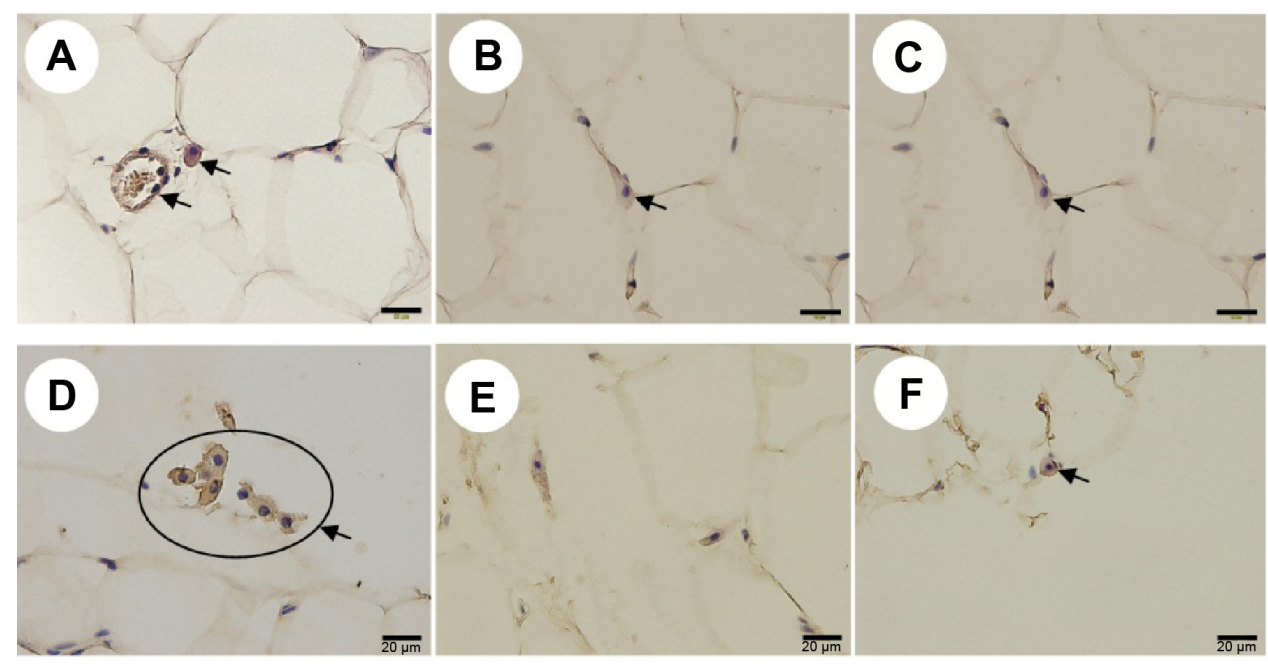

Figure 5 Immunohistochemical staining of CD68-expressing neutrophils (A-C) and F4/80-expressing macrophages (D-F) in adipose tissue of rats of all groups at week 60 with long-term treatment with or without rare sugar D-psicose.

Notes: The O-P group was treated with $5 \%$ D-psicose dissolved in drinking water, where O-C and non-diabetic LETO were fed drinking water only. All the rats were supplied a standard rat diet. Arrows indicate positive-stained cells for CD68 in O-C (A), O-P (B), and LETO (C), and for F4/80 in O-C (D), O-P (E), and LETO (F). Black circle in D indicates positive-stained cells in the circle. Magnification: $\times 400$. Scale bars: $50 \mu \mathrm{m}$.

Abbreviations: LETO, Long-Evans Tokushima Otsuka; OLETF, Otsuka Long-Evans Tokushima Fatty; O-C, OLETF control; O-P, OLETF psicose. 
Among the potential mechanisms of the toxic effects of glucose on $\beta$-cell function is chronic oxidative stress because glucose can generate ROS, ${ }^{33}$ which has adverse effects on islet function. ${ }^{23,34}$ Moreover, pancreas contains trace amounts of antioxidant enzymes and thus $\beta$-cells become liable to be injured by oxidative stress. Elevation in GSH level and super oxide dismutase may compensate oxidative stress. Studies have shown D-psicose as a strong antioxidant through the scavenging of ROS and expression of antioxidant enzymes. ${ }^{35-37}$ D-Psicose, in the present study, significantly restored the antioxidant GSH (Figure 4K), consistent with an earlier report, ${ }^{38}$ although we were unable to detect super oxide dismutase. Control of blood glucose level remains the gold standard in the prevention and management of diabetic complications. ${ }^{39}$ Treatment of OLETF rats with D-psicose was found to control blood glucose levels uniquely till the end of 60 weeks (Figure 1A). The oral glucose tolerance test also revealed that the glucose tolerance was improved (Figure 1C-E) with the significant restoration of insulin (Figure 1F-H) secretion after the glucose load. Insulin resistance initially leads to hyperinsulinemia, ${ }^{40}$ followed by hypoinsulinemia when $\beta$-cell volume is reduced through ROS-induced injury. Consistently, the present data showing initial hyperinsulinemia and hypoinsulinemia afterward suggest $\beta$-cell proliferation followed by damage in the control rats. D-Psicose treatment prevented $\beta$-cell injury through its antioxidative effects and thus improved glucose tolerance, consistent with Lee et al's findings in OLETF rats. ${ }^{41}$ Moreover, glycated hemoglobin $\mathrm{A}_{1 \mathrm{c}}$ is considered another gold standard in monitoring glucose control in view of the distinct relation between glucose and $\mathrm{A}_{1 \mathrm{c}}$ in both fasting and postprandial periods. ${ }^{42,43} \mathrm{D}$-Psicose was able to reduce $\mathrm{HbA}_{1 \mathrm{c}}$ level (Figure 1M) through maintaining normoglycemia during both fasting (Figure 1A) and postprandial (Figure 1L) periods. Moreover, postprandial hyperglycemia itself is hazardous for vascular diseases and thus considered as a predictive tool for myocardial infarction and mortality. ${ }^{44}$ Vascular diseases are mentioned as crucial results of elevated lipoproteins which may also affect $\beta$-cell survival and function. ${ }^{45}$ Our study confirmed the antihyperlipidemic effect of D-psicose with significant decrease in serum LDL (Figure 3F), although the protective HDL levels remained static all through (Figure 3E). Studies have also demonstrated that insulin resistance is a predisposing factor for dyslipidemia ${ }^{46}$ and that diabetic dyslipidemia is typically marked by elevated TC, TG, and LDL and decreased HDL, and these constitute an important risk factor for T2DM. ${ }^{47} \mathrm{D}-\mathrm{Psicose}$ treatment also resulted in significant decreases in plasma TC, TG, and LDL levels when compared to those in control (Figure 3A and B).
The presented data strongly suggest the potential of D-psicose in the commencement of diabetes rather than being a "remedy" after diabetes is already established. Although we have not tested this part, further investigation remains on process to clarify this issue. However, our results demonstrated that prolonged treatment with the rare sugar D-psicose maintained blood glucose levels, lipid profile with the amelioration of oxidative stress, and proinflammatory cytokines in T2DM in OLETF rats.

Finally, in addition to making functional foods, D-psicose might be used as a combination drug, not only for antihyperglycemic effect but also to counter some side effects marked by the chronic use of antidiabetic agents.

\section{Acknowledgments}

We are very grateful to Professor Ken Izumori for supplying D-psicose from the Rare Sugar Research Center, Kagawa University. We thank Arif-Ul-Hasan for technical assistance and Teruyoshi Izumi for animal care.

\section{Disclosure}

The authors report no conflicts of interest in this work.

\section{References}

1. Larsson B, Svardsudd K, Welin L, Wilhelmsen L, Bjorntorp P, Tibblin G. Abdominal adipose tissue distribution, obesity, and risk of cardiovascular disease and death: 13 year follow up of participants in the study of men born in 1913. Br Med J (Clin Res Ed). 1984;88:1401-1404.

2. Rahier J, Guiot Y, Goebbels RM, Sempoux C, Henquin JC. Pancreatic $\beta$-cell mass in European subjects with type 2 diabetes. Diabetes Obes Metab. 2008;10(suppl 4):32-42.

3. Koenen TB, Stienstra R, van Tits LJ, et al. Hyperglycemia activates caspase-1 and TXNIP-mediated IL-1b transcription in human adipose tissue. Diabetes. 2011;60:517-524.

4. Dandona P, Aljada A, Bandyopadhyay A. Inflammation: the link between insulin resistance, obesity and diabetes. Trends Immunol. 2004; 25:4-7.

5. Ohkita M, Kiso Y, Matsumura Y. Pharmacology in health foods: improvement of vascular endothelial function by French maritime pine bark extract (Flavangenol). J Pharmacol Sci. 2011;115:461-465.

6. Du H, van der A DL, Boshuizen HC, et al. Dietary fiber and subsequent changes in body weight and weight circumference in European men and women. Am J Clin Nutr. 2009;91:329-336.

7. Bell SJ, Goodrick GK. A functional food product for the management of weight. Crit Rev Food Sci Nutr. 2002;42:163-178.

8. Izumori K. Izumoring: a strategy for bioproduction of all hexoses. J Biotechnol. 2006;124:717-722.

9. Matsuo T, Suzuki H, Hashiguchi M, Izumori K. D-Psicose is a rare sugar that provides no energy to growing rats. J Nutr Sci Vitaminol. 2002;48:77-80.

10. Matsuo T, Izumori K. D-psicose inhibits intestinal $\alpha$-glucosidase and suppresses glycemic response after carbohydrate ingestion in rats. Tech Bull Fac Agric Kag Univ. 2006;58:27-32.

11. Baek SH, Park SJ, Lee HG. D-psicose, a sweet monosaccharide, ameliorates hyperglycemia, and dyslipidemia in C57BL/6J db/db mice. J Food Sci. 2010;75:H49-H53. 
12. Hossain MA, Kitagaki S, Nakano D, et al. Rare sugar D-psicose improves insulin sensitivity and glucose tolerance in type 2 diabetes Otsuka long-evans Tokushima fatty (OLETF) rats. Biochem Biophys Res Commun. 2011;405:7-12.

13. Hossain A, Yamaguchi F, Matsunaga T, et al. Rare sugar D-psicose protects pancreas $\beta$-islets and thus improves insulin resistance in OLETF rats. Biochem Biophys Res Commun. 2012;425:717-723.

14. Iida T, Kishimoto Y, Yoshikawa Y, et al. Acute D-psicose administration decreases the glycemic responses to an oral maltodextrin tolerance test in normal adults. J Nutr Sci Vitaminol. 2008;54:511-514.

15. Hayashi N, Iida T, Yamada T, et al. Study on the postprandial blood glucose suppression effect of D-psicose in borderline diabetes and the safety of long-term ingestion by normal human subjects. Biosci Biotechnol Biochem. 2010;74:510-519.

16. Hishiike T, Ogawa M, Hayakawa S, et al. Transepithelial transports of rare sugar D-psicose in human intestine. J Agric Food Chem. 2013;61: 7381-7386.

17. Toyoda Y, Ito Y, Tanigawa K, Miwa I. Impairment of glucokinase translocation in cultured hepatocytes from OLETF and GK rats, animal models of type 2 diabetes. Arch Histol Cytol. 2000;63:243-248.

18. Matsuo T, Baba Y, Hashiguchi M, Takeshita K, Izumori K, Suzuki H. Dietary D-psicose, a C-3 epimer of D-fructose, suppresses the activity of hepatic lipogenic enzymes in rats. Asia Pac J Clin Nutr. 2001; 10:233-237.

19. Mu W, Zhang W, Feng Y, Bo J, Zhou L. Recent advances on applications and biotechnological production of D-psicose. Appl Microbiol Biotechnol. 2012;94:1461-1467.

20. Kawano K, Hirashima T, Mori S, Saitoh Y, Kurosumi M, Natori T. Spontaneous long-term hyperglycemic rat with diabetic complications. Otsuka Long-Evans Tokushima Fatty (OLETF) strain. Diabetes 1992;41:1422-1428.

21. Butler AE, Janson J, Bonner-Weir S, Ritzel R, Rizza RA, Butler PC. $\beta$-cell deficit and increased $\beta$-cell apoptosis in humans with type 2 diabetes. Diabetes. 2003;102-110.

22. Moon JR, Smith AE, Tobkin SE, et al. Total body water changes after an exercise intervention tracked using bioimpedance spectroscopy: a deuterium oxide comparison. Clin Nutr. 2009;28:516-525.

23. Matthews DR, Hosker JP, Rudenski AS, Naylor BA, Treacher DF, Turner RC. Homeostasis model assessment: insulin resistance and beta cell function from fasting plasma glucose and insulin concentrations in man. Diabetologia. 1985;28:412-419.

24. Robertson RP, Harmon J, Tran PO, Poitout V. Beta-cell glucose toxicity, lipotoxicity, and chronic oxidative stress in type 2 diabetes. Diabetes. 2004;53(suppl 1):S119-S124.

25. Mohamed-Ali V, Goodrick S, Rawesh A, et al. Subcutaneous adipose tissue releases interleukin-6 but not tumor necrosis factor-alpha, in vivo. J Clin Endocrinol Metab. 1997;82:4196-4200.

26. Muller S, Martin S, Koenig W, et al. Impaired glucose tolerance is associated with increased serum concentrations of interleukin 6 and co-regulated acute-phase proteins but not TNF-alpha or its receptors. Diabetologia. 2002;45:805-812.

27. Zhang Y, Proenca R, Maffei M, Barone M, Leopold L, Friedman JM. Positional cloning of the mouse obese gene and its human homologue. Nature. 1994;372:425-432.

28. Moller DE, Berger JP. Role of PPARs in the regulation of obesity-related insulin sensitivity and inflammation. Int J Obes Relat Metab Disord. 2003;27:S17-S21.
29. Hummasti S, Hotamisligil GS. Endoplasmic reticulum stress and inflammation in obesity and diabetes. Circ Res. 2010;107:579-591.

30. Gregor MF, Hotamisligil GS. Inflammatory mechanisms in obesity. Annu Rev Immunol. 2011;29:415-445.

31. Bosello O, Zamboni M. Visceral obesity and the metabolic syndrome. Obes Rev. 2000;1:47-56.

32. Cancello R, Henegar C, Viguerie N, et al. Reduction of macrophage infiltration and chemoattractant gene expression changes in white adipose tissue of morbidly obese subjects after surgery-induced weight loss. Diabetes. 2005;54:2277-2286.

33. Otani M, Yamamoto M, Harada M, Otsuki M. Effect of long- and short-term treatments with pravastatin on diabetes mellitus and pancreatic fibrosis in the Otsuka-Long-Evans-Tokushima Fatty rat. Br J Pharmacol. 2010;159:462-473.

34. Donath MY, Boni-Schnetzler M, Ellingsgaard H, Ehses JA. Islet inflammation impairs the pancreatic beta-cell in type 2 diabetes. Physiology. 2009;24:325-331.

35. Murata A, Sekiya K, Watanabe Y, et al. A novel inhibitory effect of D-allose on production of reactive oxygen species from neutrophils. J Biosci Bioeng. 2003;96:89-91.

36. Murao K, Yu X, Cao WM, et al. D-psicose inhibits the expression of MCP-1 induced by high-glucose stimulation in HUVECs. Life Sci. 2007;26:592-599.

37. Suna S, Yamaguchi F, Kimura S, Tokuda M, Jitsunari F. Preventive effect of D-psicose, one of rare ketohexoses, on di-(2-ethylhexyl) phthalate (DEHP)induced testicular injury in rat. Toxicol Lett. 2007;173:107-117.

38. Aksoy N, Vural H, Sabuncu T, Alsoy S. Effects of melatonin on oxidative-antioxidative status of tissues in streptozotocin-induced diabetic rats. Cell Biochem Funct. 2003;21:121-125.

39. Bavarva JH, Narasimhacharya AV. Antihyperglycemic and hypolipidemic effects of Costus speciosus in alloxan-induced diabetic rats. Phytother Res. 2008;22:620-626.

40. Reaven GM. Banting lecture 1988. Role of insulin in human disease. Diabetes. 1988;37:1595-1607.

41. Lee E, Ryu GR, Ko SH, et al. Antioxidant treatment may protect pancreatic beta cells through the attenuation of islet fibrosis in an animal model of type 2 diabetes. Biochem Biophys Res Commun. 2011;414:397-402.

42. Woo V, Shestakova MV, Ørskov C, Ceriello A. Targets and tactics: the relative importance of $\mathrm{HbA}$, fasting and postprandial plasma glucose levels to glycaemic control in type 2 diabetes. Int J Clin Pract. 2008;62:1935-1942.

43. Avignon A, Radauceanu A, Monnier L. Nonfasting plasma glucose is a better marker of diabetic control than fasting plasma glucose in type 2 diabetes. Diabetes Care. 1997;20:1822-1826.

44. Navigator Study Group. Effect of nateglinide on the incidence of diabetes and cardiovascular events. N Engl J Med. 2010;362:1463-1476.

45. Roehrich ME, Mooser V, Lenain V, et al. Insulin-secreting beta-cell dysfunction induced by human lipoproteins. J Biol Chem. 2003;278: $18368-18375$

46. Taskinen MR. Diabetic dyslipidaemia: from basic research to clinical practice. Diabetologia. 2003;46:733-749.

47. Shin A, Sung J, Shin H, Kim J. Dietary intake, eating habits, and metabolic syndrome in Korean men. J Am Diet Assoc. 2009;109:633-640.
Drug Design, Development and Therapy

\section{Publish your work in this journal}

Drug Design, Development and Therapy is an international, peerreviewed open-access journal that spans the spectrum of drug design and development through to clinical applications. Clinical outcomes, patient safety, and programs for the development and effective, safe, and sustained use of medicines are a feature of the journal, which

\section{Dovepress}

has also been accepted for indexing on PubMed Central. The manuscript management system is completely online and includes a very quick and fair peer-review system, which is all easy to use. Visit http://www.dovepress.com/testimonials.php to read real quotes from published authors. 